Disclosure of Interests: : Serdal Ugurlu: None declared, Philip N Hawkins: None declared, Charalampia Papadopoulou: None declared, Tamer Rezk: None declared, Dorota Rowczenio: None declared, Helen J. Lachmann Grant/research support from: SOBI, Novartis, Consultant for: Novartis, Takeda, Speakers bureau: SOBI. Novartis DOI: 10.1136/annrheumdis-2019-eular.2663

\section{FRI0619 INPATIENT BURDEN AND COMORBIDITIES OF SARCOIDOSIS: NATIONWIDE INPATIENT SAMPLE 2013- 2014}

Patompong Ungprasert ${ }^{1}$, Karn Wijarnpreecha. ${ }^{2}$, Wisit Cheungpasitporn ${ }^{3}$, Charat Thongprayoon ${ }^{4}$, Paul Kroner ${ }^{2} .{ }^{1}$ Faculty of Medicine Siriraj Hospital, Mahidol University, Clinical epidemiology unit, Bangkok, Thailand: ${ }^{2}$ Mayo Clinic Florida, Jacksonville, United States of America; ${ }^{3}$ University of Mississippi Medical Center, Jackson, United States of America; ${ }^{4}$ Mayo Clinic Rochester, Rochester, United States of America

Background: Little is known about the inpatient burden and healthcare utilization among patients with sarcoidosis. Previous studies have focused on trends of hospitalization rate of patients with sarcoidosis over time [1] but none has investigated their inpatient prevalence, mortality and expenditures.

Objectives: The current study was conducted with the aims to shed more light on those characteristics as well as to investigate the comorbidities of sarcoidosis using the data from a large national database.

Methods: Patients with sarcoidosis were identified within the Nationwide Inpatient Sample (NIS) database of the years 2013 and 2014 using the ICD-9 diagnostic code. NIS is the largest publicly available inpatient database in the US. Data for more than seven million individual hospitalization across all-payers in the United States (US) is recorded annually in the NIS database. This is itself a $20 \%$ stratified sample of over 4,000 non-federal acute care hospitals from more than 40 states of the US and is representative of $95 \%$ of hospital discharges nationwide. Data on patient and hospital characteristics, comorbidities, total hospital costs and total hospitalization charges was collected. A propensity-matched cohort of patients without sarcoidosis from the same database was created and used as comparators for the analysis of comorbidities. Inpateint prevalence of sarcoidosis was calculated using all admissions in the NIS database as denominator. Odds ratios (OR) comapring the prevalence of comorbities between cases with sarcoidosis and propensity-matched controls without sarcoidosis were calculated.

Results: A cohort 78,055 patients with sarcoidosis was identified from the database, corresponding to an inpatient prevalence of 2.21 cases per 1,000 admissions. The most common reasons for admission among patients with sarcoidosis in this cohort were as follows; pneumonia $(34.3 \%)$, respiratory failure $(26.1 \%)$, cardiomyopathy $(10.9 \%)$, coronary artery disease $(9.2 \%)$, acute kidney injury $(8.5 \%)$ and atrial fibrillation $(5.5 \%)$. Analysis of comorbidities found that patients with sarcoidosis had significantly higher odds of atrial fibrillation, conduction abnormalities, aortic valvulopathy, congestive heart failure and cardiomyopathy compared to propensity-matched patients without sarcoidosis (table 1). After adjusting for confounders, patients with sarcoidosis displayed a mean additional $\$ 27,205(p<0.01)$ for total hospitalization charges (the amount of money that each hospital billed for providing its service on each case) when compared to hospitalization of patients without sarcoidosis.

Conclusion: The inpatient prevalence of sarcoidosis was relatively high compared to its overall incidence. Hospitalization of patients with sarcoidosis was associated with a significantly higher total hospitalization charges. Patients with sarcoidosis have higher risk of several cardiac comorbidities.

\section{REFERENCES:}

[1] Foreman MG, et al. (2006) Hospitalization for patients with sarcoidosis: 1979-2000. Sarcoidosis Vasc Diffuse Lung Dis 23:124-129.

Table 1. Odds ratio comparing comorbidities between patients with sarcoidosis and patients without sarcoidosis

\begin{tabular}{lccc}
\hline Variable & $\begin{array}{c}\text { Adjusted odds } \\
\text { ratio }\end{array}$ & $\begin{array}{c}95 \% \text { Confidence } \\
\text { Interval }\end{array}$ & $\begin{array}{c}\mathbf{p}- \\
\text { value }\end{array}$ \\
\hline $\begin{array}{l}\text { Atrial fibrillation } \\
\text { Conduction abnormalities } \\
\text { (blocks) }\end{array}$ & 1.41 & $1.13-1.76$ & $<0.01$ \\
Coronary artery disease & 2.04 & $1.45-2.89$ & $<0.01$ \\
Congestive heart failure & 1.10 & & \\
Cardiomyopathy & 1.23 & $0.94-1.29$ & 0.26 \\
Aortic valvulopathy & 1.25 & $1.04-1.45$ & 0.02 \\
\hline
\end{tabular}

Disclosure of Interests: None declared

DOI: 10.1136/annrheumdis-2019-eular.514

\section{FRI0620 FMF PATIENTS HAVE A HIGHER PREVALENCE OF SPA-RELATED DISORDERS INCLUDING MHC-I- OPATHIES THAN CONTROLS: INSIGHTS FROM A LARGE COHORT STUDY}

Abdulla Watad $^{1}$, Nicola Luigi Bragazzi ${ }^{2}$, Dennis Mcgonagle ${ }^{3}$, Doron Comanesther ${ }^{4}$, Arnon Cohen ${ }^{5}$, Howard Amital'. ' 'Sheba Medical Center, Internal Medicine B, Tel Aviv, Israel; ${ }^{2}$ University of Genoa, Genoa, Italy, Postgraduate School of Public Health, Department of Health Sciences (DISSAL), Genoa, Italy, ${ }^{3}$ University of Leeds, NIHR Leeds Musculoskeletal Biomedical Research Unit, Chapel Allerton Hospital, Leeds, UK, Section of Musculoskeletal Disease, Leeds Institute of Molecular Medicine, Leeds, United Kingdom; ${ }^{4}$ Clalit Health Service, Epidemiology, Tel Aviv, Israel; ${ }^{5}$ Clalit Health Service, Epidemiology, Tel Aviv, Israel

Background: Familial Mediterranean fever (FMF) is a common, hereditary autoinflammatory disorder, caused by mutations in the MEFV gene encod ing for pyrin. MEFV mutations have been also reported in spondyloarthritis ( $\mathrm{SpA}$ )-associated disorders including Crohn's disease, ulcerative colitis, Behçet's disease, psoriasis and ankylosing spondylitis (AS) with the latter 3 disorders considered under the umbrella term of "MHC-I-opathies" due to population-level associations of HLA-B51, Cw0602 and HLA-B27, respectively. FMF and the $\mathrm{SpA}$ group of disorders share an association with disease localization to site of physical stress or microdamage with innate immune activation at such sites as a primary driver of immunopathology but this is not the case for classical autoimmune diseases where central tolerance failure is an overarching immunological concept.

Objectives: To test the hypothesis that autoinflammation in FMF may exaggerate the tendency towards adaptive immunopathology or $\mathrm{MHC}$ class-I associated disorders and therefore a higher prevalence of SpArelated disorders in patients with FMF.

Methods: 7,747 FMF patients and 10,080 age- and sex-matched controls in the Clalit-Health-Services medical database were identified and compared in terms of prevalence of SpA-associated disorders and also evaluated 4 classical and strong MHC class-II associated disorders, namely sarcoidosis, pernicious anaemia, pemphigus vulgaris, and myasthenia gravis to ascertain whether such associations with SpA-spectrum disease were specific or merely reflected the non-specific consequences of innate immune system activation on driving divergent types of immunity.

Results: FMF showed a strong association with MHC class I related dis eases: OR $28.58([95 \% \mathrm{Cl}$ 6.93-117.87], $\mathrm{p}<0.0001)$ for Behçet's disease OR of $10.33([95 \% \mathrm{Cl} 4.09-26.09], \mathrm{p}<0.0001)$ for $\mathrm{AS}$, and OR $1.67([95 \%$ $\mathrm{Cl} 1.19-2.33], \mathrm{p}=0.0029)$ for psoriasis. For weakly $\mathrm{MHC}$ class I linked diseases, an OR of $3.76([95 \% \mathrm{Cl} 2.48-5.69], \mathrm{p}<0.0001)$ for Crohn's disease and OR of $2.64([95 \% \mathrm{Cl} 1.52-4.56], \mathrm{p}=0.0005)$ for ulcerative colitis were found. No association was found between FMF and strongly MHC class II-associated including pemphigus vulgaris, sarcoidosis, pernicious anaemia and myasthenia gravis. At the Cox multivariate survival analysis, the mortality of FMF patients was higher only in Crohn's disease with an HR of 2.32 ([95\%Cl 1.09-4.93], $\mathrm{p}=0.0291)$

Conclusion: FMF patients are associated with increased risk of SpArelated disease diagnosis including $\mathrm{MHC}$-I-opathies suggesting that tissuespecific dysregulation of innate immunity share between FMF and SpA spectrum disorders may drive adaptive immune MHC class I associated conditions.
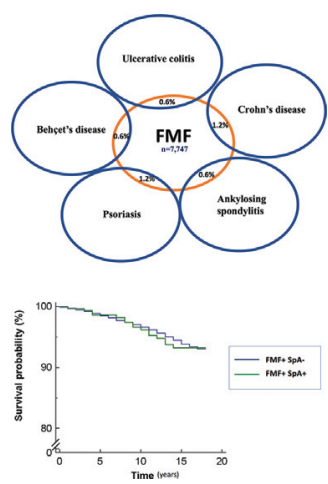

Figure 1. The proportion of FMF patients with $\mathrm{SpA}$ related disorders $(\mathrm{A})$ and their impact on FMF patients' survival (B) 
Disclosure of Interests: Abdulla Watad: None declared, Nicola Luigi Bragazzi: None declared, Dennis McGonagle Consultant for: Lilly, Novartis UCB, Speakers bureau: Lilly, Novartis UCB, Doron Comanesther: None declared, Arnon Cohen Grant/research support from: Prof. Arnon Cohen received research grants from Janssen, Novartis and AbbVie and Sanofi, Consultant for: Prof. Arnon Cohen served as a consultant, advisor for AbbVie; Amgen; Boehringer Ingelheim; Dexcel pharma; Janssen, Lilly; Neopharm; Novartis, Perrigo; Pfizer; Rafa; Sanofi, Speakers bureau: Prof. Arnon Cohen served as speaker for AbbVie; Amgen; Boehringer Ingelheim; Dexcel pharma; Janssen, Lilly; Neopharm; Novartis, Perrigo; Pfizer; Rafa; Sanofi, Howard Amital Grant/research support from: Pfizer, AbbVie, Janssen, Grant/research support from: Pfizer, AbbVie, Janssen, Consultant for: Pfizer, Merck Sharp \& Dohme, Consultant for: Pfizer, Merck Sharp \& Dohme, Speakers bureau: Pfizer, Merck Sharp \& Dohme, Janssen, Sanofi, Bristol-Myers Squibb, Abbvie, Neopharm, Speakers bureau: Pfizer, Merck Sharp \& Dohme, Janssen, Sanofi, Bristol-Myers Squibb, Abbvie, Neopharm

DOI: 10.1136/annrheumdis-2019-eular.1088

\section{FRI0621 ASSOCIATIONS BETWEEN ORGAN INVOLVEMENTS AND GENDER, ALLERGY, AND MALIGNANCY IN 166 PATIENTS WITH IGG4-RELATED DISEASE}

Hajime Yoshifuji ${ }^{1}$, Mirei Shirakashi ${ }^{1}$, Yuzo Kodama ${ }^{2}$, Tsutomu Chiba ${ }^{3}$, Motohisa Yamamoto ${ }^{4}$, Hiroki Takahashi ${ }^{4}$, Kazushige Uchida ${ }^{5}$, Kazuichi Okazaki ${ }^{5}$, Tetsuya Ito $^{6}$, Shigeyuki Kawa ${ }^{7}$, Kazunori Yamada ${ }^{8}$, Mitsuhiro Kawano ${ }^{8}$, Shintaro Hirata ${ }^{9}$, Yoshiya Tanaka ${ }^{10}$, Masafumi Moriyama ${ }^{11}$, Seiji Nakamura ${ }^{11}$, Terumi Kamisawa ${ }^{12}$, Shoko Matsui ${ }^{13}$, Hiroto Tsuboi ${ }^{14}$, Takayuki Sumida ${ }^{14}$, Motoko Shibata $^{15}$, Hiroshi Goto ${ }^{15}$, Yasuharu Sato ${ }^{16}$, Tadashi Yoshino ${ }^{16}$ Tsuneyo Mimori ${ }^{1}$. 'Kyoto University, Kyoto, Japan; ${ }^{2}$ Kobe University, Kobe, Japan ${ }^{3}$ Kansai Electric Power Hospital, Osaka, Japan; ${ }^{4}$ Sapporo Medical University, Sapporo, Japan; ${ }^{5}$ Kansai Medical University, Hirakata, Japan; ${ }^{6}$ Shinshu University, Matsumoto, Japan; ${ }^{7}$ Matsumoto Dental University, Shiojiri, Japan; ${ }^{8}$ Kanazawa University, Kanazawa, Japan; ${ }^{9}$ Hiroshima University, Hiroshima, Japan; ${ }^{10}$ University of Occupational and Environmental Health, Fukuoka, Japan; ${ }^{11}$ Kyushu University, Fukuoka, Japan; ${ }^{12}$ Tokyo Metropolitan Komagome Hospital, Tokyo, Japan; ${ }^{13}$ University of Toyama, Toyama, Japan; ${ }^{14}$ University of Tsukuba, Tsukuba, Japan; ${ }^{15}$ Tokyo Medical University, Tokyo, Japan; ${ }^{16}$ Okayama University,

Okayama, Japan

Background: Symptomatic differences between male and female patients with IgG4-related disease (IgG4-RD) have been reported [1]. Associations between IgG4-RD and allergy and malignancy have also been reported, although they are controversial.

Objectives: We previously analyzed the treatment course of IgG4-RD using a multi-center cohort [2]. In the present study, we investigated the associations between organ symptoms and gender, allergic diseases, and malignancies.

Methods: Data of 166 patients with a definitive diagnosis of IgG4-RD were retrospectively analyzed.

Results: The cohort consisted of 108 men (65\%) and 58 women (35\%). Most frequently, the submandibular gland $(52 \%)$, pancreas $(46 \%)$, lachrymal gland $(43 \%)$, and lymph node $(38 \%)$ were affected. Furthermore, we assessed the frequencies of female sex, allergy, and malignancy in patients with each affected organ (Table 1). The organs with high frequencies of women included the lachrymal gland (50\%), lungs (44\%), and submandibular gland $(43 \%)$. Those with low frequencies of women included the thyroid gland $(0 \%)$, retroperitoneum $(17 \%)$, and para-aorta (19\%). In addition, allergic diseases were found in 60 patients $(36 \%)$ (Fig. 1A). The organs with high frequencies of allergy included the thyroid gland $(50 \%)$, submandibular gland $(47 \%)$, and lachrymal gland $(40 \%)$. Those with low frequencies of allergy were the para-aorta $(13 \%)$, prostate gland $(30 \%)$, and retroperitoneum (31\%). Malignancies were found in 23 (14\%) patients (Fig. 1B). Organs with high frequencies of malignancy included the para-aorta (38\%), prostate gland $(30 \%)$, and retroperitoneum $(21 \%)$. In comparison, those with low frequencies of malignancy included the thyroid gland $(0 \%)$, lungs $(8 \%)$, and kidney $(8 \%)$.

Conclusion: Craniocervical organ involvement was associated with female sex and presence of allergic diseases, suggesting that allergy may contribute to onset of IgG4-RD in some patients. Abdominal organ involvement was associated with male sex and presence of malignancies, suggesting that malignancy may in part contribute to IgG4-RD.
REFERENCE:

[1] Wang, et al., Rheumatology, 2018. [2] Shirakashi, et al., Sci Rep, 2018.

Table 1. Frequencies of female sex, allergy, and malignancy in patients with indicated affected organ.

\begin{tabular}{|c|c|c|c|c|c|}
\hline & $\begin{array}{c}\text { Women } \\
(\%)\end{array}$ & *1 & $\begin{array}{c}\text { Allergy } \\
(\%)\end{array}$ & *2 & $\begin{array}{c}\text { Malignancy } \\
(\%)\end{array}$ \\
\hline Eye orbit & 23 & $\mathrm{~L}$ & 38 & $\mathbf{H}$ & 15 \\
\hline Lachrymal gl. & 50 & $\mathbf{H}$ & 40 & H & 11 \\
\hline Parotid gl. & 36 & $\mathbf{H}$ & 36 & - & 18 \\
\hline $\begin{array}{l}\text { Submandibular } \\
\text { gl. }\end{array}$ & 43 & $\mathbf{H}$ & 47 & $\mathbf{H}$ & 13 \\
\hline Thyroid gl. & 0 & $\mathrm{~L}$ & 50 & $\mathbf{H}$ & 0 \\
\hline Lung & 44 & $\mathbf{H}$ & 32 & $\mathrm{~L}$ & 8 \\
\hline Pancreas & 31 & $\mathrm{~L}$ & 32 & $\mathrm{~L}$ & 18 \\
\hline Bile duct & 23 & $\mathrm{~L}$ & 33 & $\mathrm{~L}$ & 15 \\
\hline Kidney & 40 & $\mathbf{H}$ & 36 & - & 8 \\
\hline Retroperitoneum & 17 & $\mathrm{~L}$ & 31 & $\mathrm{~L}$ & 21 \\
\hline Para-aorta & 19 & $\mathrm{~L}$ & 13 & $\mathrm{~L}$ & 38 \\
\hline Prostate gl. & 0 & $\mathrm{~L}$ & 30 & $\mathrm{~L}$ & 30 \\
\hline Lymph node & 41 & $\mathbf{H}$ & 38 & $\mathbf{H}$ & 13 \\
\hline Total & 35 & - & 36 & - & 14 \\
\hline
\end{tabular}

${ }^{*} \mathrm{H}, \mathrm{L}$, or "-" are marked if the frequency is higher, lower, or no change respectively, when compared to the total frequencies of female sex $(35 \%)^{1 *}$, allergy $(36 \%)^{2 *}$, and malignancy $(14 \%)^{3 *}$.
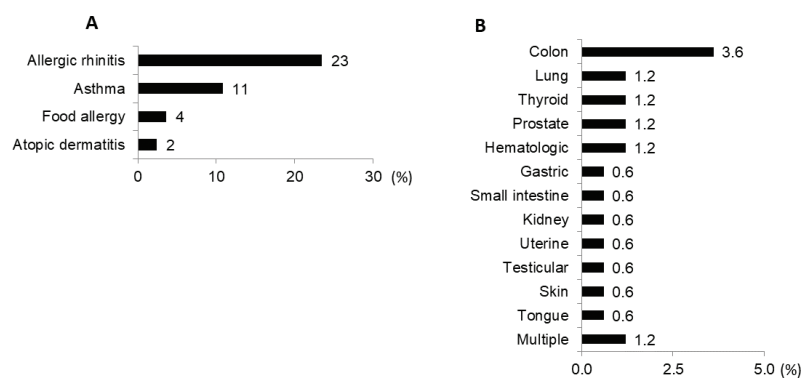

Figure 1. Frequency of allergic diseases (A) and malignancies (B) in 166 patients with IgG4-RD.

Disclosure of Interests: Hajime Yoshifuji: None declared, Mirei Shirakashi: None declared, Yuzo Kodama: None declared, Tsutomu Chiba: None declared, Motohisa Yamamoto: None declared, Hiroki Takahashi: None declared, Kazushige Uchida: None declared, Kazuichi Okazaki: None declared, Tetsuya Ito: None declared, Shigeyuki Kawa: None declared, Kazunori Yamada: None declared, Mitsuhiro Kawano: None declared, Shintaro Hirata Grant/research support from: Eli Lilly, UCB, Consultant for: Bristol-Myers Squibb, Jansen, UCB, Paid instructor for: AbbVie, Eisai, Tanabe-Mitsubishi, Speakers bureau: AbbVie, Astellas, Ayumi, BristolMyers Squibb, Chugai, Eisai, Eli Lilly, Jansen, Kissei, Pfizer, Sanofi, Takeda, Tanabe-Mitsubishi, UCB, Yoshiya Tanaka Grant/research support from: Abbvie, Astellas, Bristol-Myers Squibb, Chugai, Daiichi-Sankyo, Eisai, Mitsubishi-Tanabe, MSD, Ono, Taisho-Toyama, Takeda, Speakers bureau: Abbvie, Asahi-kasei, Astellas, Bristol-Myers Squibb, Chugai, Daiichi-Sankyo, Eli Lilly, Eisai, Glaxo-Smithkline, Janssen, Mitsubishi-Tanabe, Novartis, Pfizer Japan Inc, Sanofi, Takeda, UCB, YL Biologics, Masafumi Moriyama: None declared, Seiji Nakamura: None declared, Terumi Kamisawa: None declared, Shoko Matsui: None declared, Hiroto Tsuboi: None declared, Takayuki Sumida Grant/research support from: Bristol-Myers Squibb, Speakers bureau: Bristol-Myers Squibb, Motoko Shibata: None declared, Hiroshi Goto: None declared, Yasuharu Sato: None declared, Tadashi Yoshino: None declared, Tsuneyo Mimori: None declared DOI: 10.1136/annrheumdis-2019-eular.1264 